\title{
Optimization of the Magnetic Properties of Materials for Fluxgate Sensors
}

\author{
Luiz, Carlos de Carvalho Benyosef, Giovanni Chaves Stael*, Maurício Bochner
}

Observatório Nacional, Rua General José Cristino, 77, 20921-400 Rio de Janeiro - RJ, Brazil

Received: April 12, 2007; Revised: April 22; 2008

\begin{abstract}
A study was made of the variation of the magnetic properties of cobalt-based alloys using different compositions of $\mathrm{CoFeSiB}$ and $\mathrm{CoFeSiBCr}$ systems, which were produced by the melt-spinning technique and some of them subjected to a stress annealing treatment. A comparative study of core geometry and supporting material was also performed in order to obtain low noise fluxgate sensor core using amorphous magnetic ribbons of these alloys. The best alloy was a stress annealed $\mathrm{Co}_{67.5} \mathrm{Fe}_{3.5} \mathrm{Si}_{17.4} \mathrm{~B}_{11.6}$ sample, which yielded fluxgate sensors with lower noise levels than those of commercial crystalline materials.
\end{abstract}

Keywords: amorphous alloys, fluxgate sensor, stress annealing, magnetic permeability

\section{Introduction}

The fluxgate sensor consists of a magnetic core made of highly permeable materials that are excited up to the saturation limit by a periodic current source with a $\mathrm{f}_{0}^{1}$ frequency. If the axis of the sensor is aligned to an external magnetic field, a tension signal containing the even harmonics of that excitement frequency ${ }^{2}$ is induced in the windings of the sensor coil. The intensity of the external field is proportional to the induced voltage signal (V), according to Equation 1:

$$
\mathrm{V}=\mathrm{n}_{\mathrm{s}} \mathrm{A} \frac{\mathrm{d} \Phi}{\mathrm{dt}}
$$

where $n_{s}$ is the number of windings of the sensor coil, $A$ is the core cross-sectional area and $\Phi$ is the magnetic flux in the coil.

It is well known that the characteristics of the core material are fundamental in improving the intensity of the induced signal, which in turn supplies information on the field to be evaluated ${ }^{3}$. However, it is advisable to use materials with high relative permeability $\left(\mu_{\mathrm{r}}\right)$ and demagnetization factor (D), as can be verified by the fundamental fluxgate equation (Equation 2):

$$
\mathrm{V}_{\mathrm{sec}}=\mathrm{n}_{\mathrm{s}} \mathrm{AB}_{\mathrm{ex}}(1-\mathrm{D}) \frac{\left(\mathrm{d} \mu_{\mathrm{r}} / \mathrm{dt}\right)}{\left[1+\mathrm{D}\left(\mu_{\mathrm{r}}-1\right)\right]^{2}}
$$

Thanks to its high versatility allied with good sensitivity, low consumption and small dimensions, the fluxgate magnetometer has widespread applications in several branches of geophysics, the most prominent ones being space research and magnetic observatories.

When this equipment was introduced at the beginning of 1930 , the sensors used ferrite type NiFe alloys. In subsequent decades, technological advances led to the replacement of these materials with other crystalline alloys of mu-metal and perm-alloy types. In the late 1980s, these alloys were substituted by cobalt-based amorphous materials. The outstanding feature of these alloys is the development of sensors with higher sensitivity, smaller dimensions and reduced noise level ${ }^{1,4}$. For example, commercial sensors currently available built with crystalline materials of toroidal geometry generate noise levels $^{2}$ of $100 \mathrm{pT}$ rms magnitude, in the interval of 0.1 to $6.0 \mathrm{~Hz}$, while the amorphous materials developed in this study have noise levels below $70 \mathrm{pT}$ rms.
Fluxgate sensor ${ }^{5}$ noise analyses have found that the noise is closely connected with the Curie temperature of the material, and that it is nonexistent at the Curie temperature $\left(\mathrm{T}_{c}\right)$. Hence, it is convenient to choose materials with a low $\mathrm{T}_{\mathrm{c}}$. The $\mathrm{T}_{\mathrm{c}}$ value can be controlled easily in amorphous alloys ${ }^{6}$ through the concentration of $\mathrm{Cr}$ and/or $\mathrm{Co}$.

Magnetostriction is one of the factors that determine the level of noise in fluxgate sensor cores. Materials with a null magnetostriction coefficient, or close to this value, produce sensors with lower noise levels than similar materials ${ }^{7,8}$. The geometry is also a decisive factor in the development of high resolution sensors. Among the existing geometries, the most qualified one is the ring-core. Sensors with this geometry offer additional advantages over those with a linear geometry. Thanks to their simple geometry, adjustments between the two semi-cores can be made easily during their assembly, reducing the noise level and increasing the sensitivity. In addition, some works ${ }^{9-11}$ have shown the existence of a dependence between the process of magnetization of amorphous ribbon and the effects induced by the application of an external load ("stress") on the ribbon during the adjustment process in the core. However, few studies have examined the effects of these external stresses on the noise level of sensors.

It is clear that metallic glasses present interesting properties. They are attractive for practical uses in structural and functional applications in different systems ${ }^{12}$.

\section{Experimental}

The choice of the alloys used in this work was based on the magnetic properties expected for the development of a sensor providing high sensitivity with a low noise level. Thus, compositions were selected which have low values of coercive force, Curie temperature and near zero magnetostriction, besides selecting options to obtain high values of magnetic permeability ${ }^{3}$. Three alloys of different compositions were obtained: Alloy $1\left(\mathrm{Co}_{67.5} \mathrm{Fe}_{3.5} \mathrm{Si}_{17.4} \mathrm{~B}_{11.6}\right)$, Alloy 2 $\left(\mathrm{Co}_{66} \mathrm{Fe}_{4} \mathrm{Si}_{15} \mathrm{~B}_{15}\right)$ and Alloy $3\left(\mathrm{Co}_{66.5} \mathrm{Fe}_{3.5} \mathrm{Si}_{12} \mathrm{~B}_{14} \mathrm{Cr}_{4}\right)$. These alloys were produced by the melt-spinning technique, which consists of ejecting the molten material onto an iron wheel rotating at an angular velocity sufficiently high to prevent the material from crystallizing ${ }^{13}$. Of the three alloys, only alloy 1 was subjected to the stress annealing heat treatment under mechanical tension. 


\subsection{The melt-spinning technique}

This is the method most commonly used in the production of good quality amorphous magnetic ribbons for the construction of fluxgate sensor cores. Its prime advantage is the dimensional uniformity of the ribbon along all its length. Alloys 2 and 3 of this work were obtained by the melt-spinning technique ${ }^{14}$, whereby the sample is melted by high frequency, in quartz crucibles, through an induction coil that raises the temperature to $1260{ }^{\circ} \mathrm{C}$. The samples are then ejected onto a copper wheel at a cooling rate of $104 \mathrm{~K} / \mathrm{s}$ and kept under a constant argon pressure of $170 \mathrm{mmHg}$. The ribbons obtained by this process have an average density of $7.85 \mathrm{~g} . \mathrm{cm}^{-3}$, mean thickness of $0.027 \mathrm{~mm}$ and width of $1.5 \mathrm{~mm}$.

Alloy 1 was produced in a commercial melt-spinner, which ejects the samples under constant pressure in a vacuum onto an iron wheel. The ribbons have an average density of $7.6 \mathrm{~g} . \mathrm{cm}^{-3}$, average thickness of $0.025 \mathrm{~mm}$ and width of $1.0 \mathrm{~mm}$.

\subsection{Stress annealing heat treatment}

A long cylindrical resistive oven was used, whose lateral extremities were practically sealed and which contained only two small openings, one at each end, for the passage of the ribbon. The ribbon was injected into the upper part of a chamber filled with argon gas for atmospheric control.

To eliminate external magnetic contributions during the treatment, the oven was positioned in a direction perpendicular to the magnetic north-south axis.

According to previous works ${ }^{5,3,15-20}$, heat treatments were chosen that improved the magnetic characteristics of the ribbons, such as the reduction of coercive force and approximation of the magnetostriction coefficient value to zero for the generation of low noise levels. The procedure employed in this work consisted of a 1-hour thermomechanical treatment at $340{ }^{\circ} \mathrm{C}$ in an argon atmosphere, under a longitudinal tension of $300 \mathrm{MPa}$. This procedure was preceded by a 1-hour internal stress relief treatment, at the same temperature and atmospheric condition.

The purpose of this annealing treatment was to form a transverse magnetic anisotropy in the magnetization axis, aligned with the ribbon's longitudinal axis. This phenomenon results from a higher intensity in the magnetic domain rotation substituting the movement of walls.

\subsection{Core assembly of fluxgate sensors}

Fluxgate sensors with ring-core geometry were built on supports of different materials. They were constructed for each sample studied here. Two magnetic cores with different diameters were used in the study of alloy 1 . The smaller core had an internal diameter of $16 \mathrm{~mm}$ and external diameter of $18 \mathrm{~mm}$, a nominal external width of $2.9 \mathrm{~mm}$ and internal width of $1.6 \mathrm{~mm}$. The larger core had an internal diameter of $25 \mathrm{~mm}$ and external diameter of $26.5 \mathrm{~mm}$. Its nominal external width was $2.9 \mathrm{~mm}$ and its internal width was $1.6 \mathrm{~mm}$. The larger diameter core was used with the three alloys, in samples B, C, $\mathrm{D}$ and $\mathrm{E}$. Each sensor-core contained ten layers of ribbon.
Part of the analysis was done in a Walker Scientific hysteresimeter (model AMH-15) for soft magnetic materials, and the remainder in a hysteresimeter developed by Magnetic Sensor Development Laboratory from the National Observatory.

\section{Results and Discussions}

\subsection{Influence of diameter variation and material type of the support on the magnetic properties}

Table 1 lists the coercive force values of samples A and B tested through the frequency variation experiment. These samples of the same alloy but with different internal diameters of the support, 16 and $25 \mathrm{~mm}$, respectively, were stress annealed in order to induce a magnetic anisotropy in the material, promoting a more intense domain rotation than wall movement, thus rendering the magnetic material more permeable. Table 1 indicates that there is a meaningless variation in $\mathrm{H}_{\mathrm{c}}$ values for both samples in the same frequency, in spite of the curvature radius imposed on each of them (involving different stresses). However, note the increase in the values of $\mathrm{H}_{c}$ with increased frequency.

Table 1 lists the maximum induction or saturation values of samples A and B. All the samples displayed practically constant values during the frequency variation experiment, showing no influence of the frequency on the induction saturation of materials. Sample B, whose support had a larger diameter, showed lower induction saturation. This difference was due to the higher internal stress of the material in sample A, generated by the smaller curvature radius imposed on the ribbon. The higher value of induction saturation in sample A also led to an increase in the energy loss by hysteresis and eddy currents, as can be seen in Equations 3 and 4, respectively. The constant $\mathrm{K}$ depends on the quality of iron, f represents the frequency applied, Bm the maximum induction or saturation of the samples, $\mathrm{V}$ the total volume of the group of coiled ribbons, and $\mathrm{d}$ is the ribbon thickness.

$$
\begin{aligned}
& \mathrm{Ph}=\mathrm{K} \cdot \mathrm{f} \cdot \mathrm{Bm}^{2} \\
& \mathrm{Pf}=\mathrm{K} \cdot \mathrm{d}^{2} \cdot \mathrm{f}^{2} \cdot \mathrm{Bm}^{2} \cdot \mathrm{V}
\end{aligned}
$$

Figure 1 illustrates the difference in the inclination of the hysteresis curves of samples A and B (stress annealed) with diameters of $16 \mathrm{~mm}$ and $25 \mathrm{~mm}$ respectively, at a frequency of $10 \mathrm{kHz}$.

The higher inclination in the curve of sample $\mathrm{B}$ indicates the formation of a transverse magnetic anisotropy in the difficult axis of magnetization, aligned with the longitudinal axis of the ribbon, which is more pronounced than in sample A. This phenomenon results from a highly intense rotation of the domains instead of wall movements. The higher stress observed in sample A prevents a marked rotation of magnetic domains and, hence, of the hysteresis curve, making the material less suitable for fluxgate magnetometers.

The determination of the remanent induction $(\mathrm{Br})$ of the treated samples A and B with different internal diameters revealed a very slow growth of induction values throughout the frequency range

Table 1. Values of $\mathrm{Hc}(\mathrm{Oe})$ and $\mathrm{Bmax}(\mathrm{kG})$ resulting from frequency variation imposed on samples A and $\mathrm{B}$.

\begin{tabular}{lllllll}
\hline & Samples & $0.5 \mathrm{kHz}$ & $1 \mathrm{kHz}$ & $5 \mathrm{kHz}$ & $7.5 \mathrm{kHz}$ & $10 \mathrm{kHz}$ \\
\hline A (alloy 1) & $\mathrm{H}_{\mathrm{c}}(\mathrm{Oe})$ & 0.0348 & 0.0464 & 0.1155 & 0.1407 & 0.1709 \\
& $\mathrm{Bmax}(\mathrm{kG})$ & 4.1 & 4.2 & 4.1 & 4.2 & 4.1 \\
B (alloy 1) & $\mathrm{H}_{\mathrm{c}}(\mathrm{Oe})$ & 0.0451 & 0.0545 & 0.1071 & 0.1252 & 0.1949 \\
& $\operatorname{Bmax}(\mathrm{kG})$ & 2.6 & 2.6 & 2.5 & 2.5 & 2.6 \\
\hline
\end{tabular}




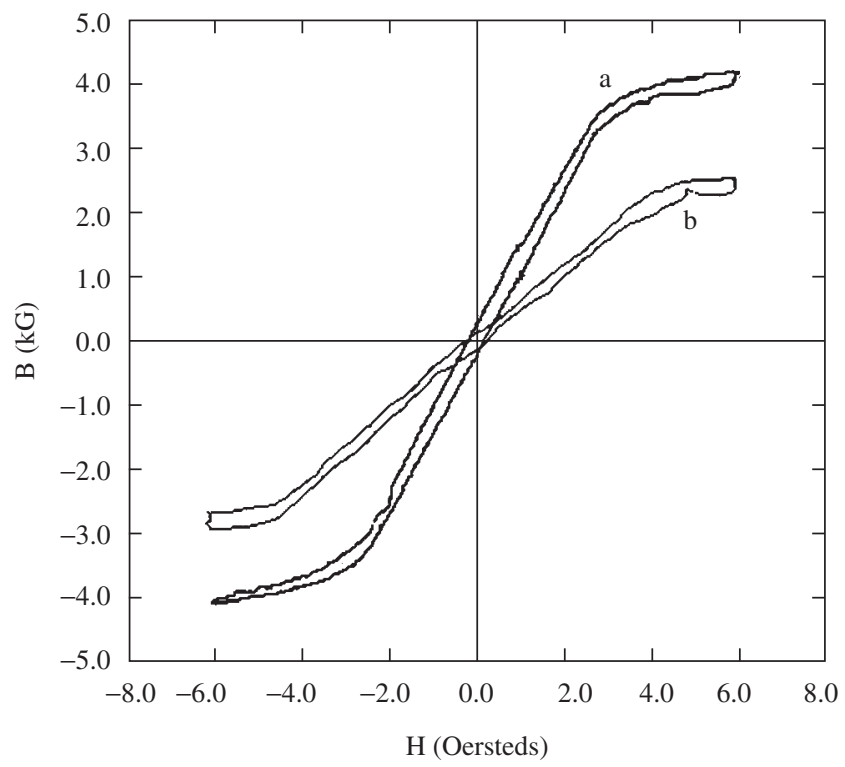

Figure 1. Hysteresis curves of stress annealed samples a and $\mathrm{b}$ with diameters of 16 and $25 \mathrm{~mm}$, respectively, at a frequency of $10 \mathrm{kHz}$.

of 0.1 to $0.2 \mathrm{kG}$. Moreover, no significant variation of $\mathrm{Br}$ was found in either of the samples at the same frequency.

Table 2 shows the magnetic properties of samples $C$ and $D$ in the untreated ("as-cast") condition, measured at a constant frequency of $10 \mathrm{kHz}$, with Nylon and Titanium supports, respectively.

The magnetic properties of samples $\mathrm{C}$ and $\mathrm{D}$ at the same frequency did not show significant differences. The same behavior was observed with frequency variation. Therefore, it was concluded that all of the materials under study can be used as supports in the preparation of fluxgate magnetometer cores, without major variations in their performance.

Figure 2 shows the $\mathrm{B} \times \mathrm{H}$ graphs of the magnetic behavior of alloy 2 under an alternate field in the as-cast condition in samples $\mathrm{C}$ and D.

In Figure 2, note the two very similar curves in the square form and in the area of energy loss by hysteresis cycles, which is congruent with the values of magnetic properties listed in Table 2 for untreated materials and in strong contrast with the slope of the hysteresis curves of samples A and B after stress annealing.

\subsection{Analysis of the influence of alloy type and ribbon width on magnetic properties}

Table 2 lists the magnetic properties of samples $\mathrm{C}\left(\mathrm{Co}_{66} \mathrm{Fe}_{4} \mathrm{Si}_{15} \mathrm{~B}_{15}\right)$ and $\mathrm{E}\left(\mathrm{Co}_{66.5} \mathrm{Fe}_{3.5} \mathrm{Si}_{12} \mathrm{~B}_{14} \mathrm{Cr}_{4}\right)$ in the as-cast condition, with the same support diameters, at a constant frequency of $10 \mathrm{kHz}$, and different chemical compositions and ribbon widths.

A comparison of the untreated samples $\mathrm{C}$ and $\mathrm{E}$ indicates that, at a frequency of $500 \mathrm{~Hz}$, these two alloys displayed similar coercive values. However, when the frequency increased, the coercivity of alloy 2 increased in relation to that of alloy 3 , indicating greater permeability and lower energy loss in alloy 3 . This difference was attributed mainly to the greater width of alloy 2 (sample C), which presented a larger volume for the passage of Foucault currents, leading to a higher energy loss by the Joule effect in its layers.

A comparison of the values of saturation induction or maximum induction in the untreated samples $\mathrm{C}$ and $\mathrm{E}$ reveals a $62 \%$ increase in sample $\mathrm{C}$ in relation to sample $\mathrm{E}$. Again, this was due to the influence of the larger width of the sample $\mathrm{C}$ ribbon, which allowed for

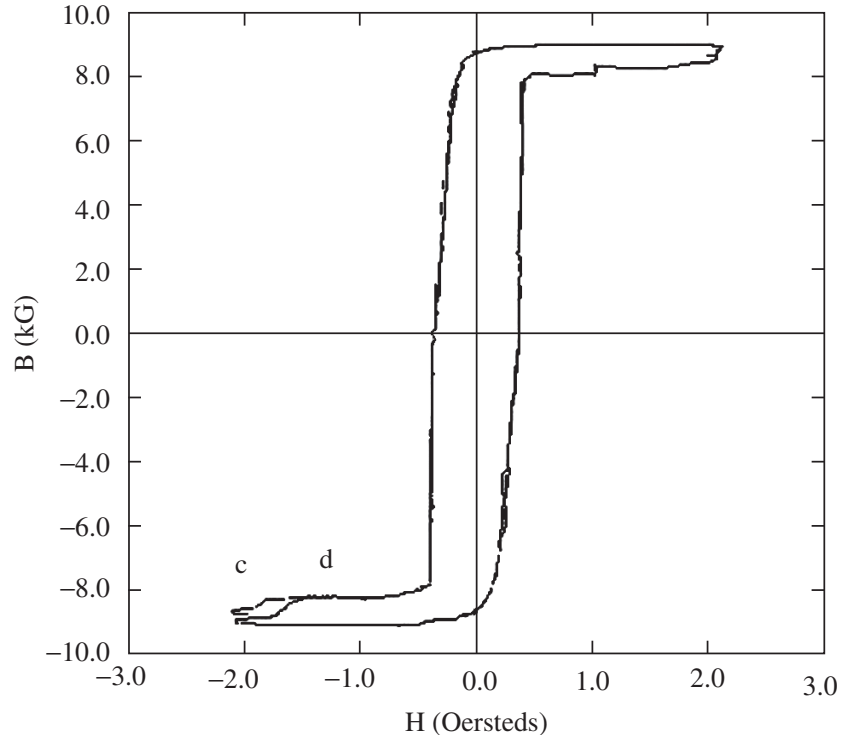

Figure 2. Hysteresis curves of the as-cast samples c and d. with Nylon and Titanium supports, respectively, at a frequency of $10 \mathrm{kHz}$

Table 2. Magnetic properties of samples A, B, C, D and E in the as-cast and stress annealed conditions, with $\mathrm{f}=10 \mathrm{kHz}$.

\begin{tabular}{ccccc}
\hline Samples & Hc $(\mathrm{Oe})$ & $\mathrm{Bs}(\mathrm{kG})$ & $\mathrm{Br}(\mathrm{kG})$ & $\mathrm{Wh}\left(\mathrm{J} / \mathrm{m}^{3}\right)$ \\
\hline A (alloy 1) & 0.1709 & 4.1 & 0.2 & 25.83 \\
B (alloy 1) & 0.1949 & 2.6 & 0.2 & 20.46 \\
C (alloy 2) & 0.3547 & 9.1 & 8.7 & 104.17 \\
D (alloy 2) & 0.3564 & 8.8 & 8.5 & 101.76 \\
E (alloy 3) & 0.2336 & 5.6 & 4.3 & 40.99 \\
\hline
\end{tabular}

the passage of larger amounts of eddy currents, thereby increasing the width of the hysteresis curve.

The samples showed strong differences in terms of remanent induction. Sample E, which had a smaller ribbon width and contained additional chromium, showed a 53\% lower remanent induction than sample $\mathrm{C}$ at the applied frequency. Once again, the larger width of the ribbon in sample $\mathrm{C}$ caused the material to undergo a larger loss of energy by the Joule effect and increased its remanence.

The magnetic properties of the samples displayed quite a significant difference, mainly due to the smaller width of alloy 3 , which presented low values of energy loss for hysteresis. The characteristics of this difference in the untreated samples $\mathrm{C}$ and $\mathrm{E}$ are illustrated by the hysteresis curves in Figure 3, at a constant frequency of $10 \mathrm{kHz}$.

For sample E, Figure 3 shows a hysteresis curve area smaller than that of sample $\mathrm{C}$, confirming the energy losses by the hysteresis cycle given in Table 2. Note the accentuated square shape of sample $\mathrm{C}$, which is characterized by the coinciding direction of the easy axis of magnetization with the direction of the longitudinal axis of the ribbon.

\subsection{Analysis of the influence of heat treatment on the magnetic properties}

Thermomechanical stress annealing is aimed at inducing magnetic anisotropy in the material, thereby promoting a domain rotation that is more intense than the movement of the walls during the process of magnetization with alternate fields. The preliminary stress relief treatment consisted of heating the ribbon to relieve the 
internal stresses generated in the material during the melt-spinning production process.

Figures 4 and 5 show representative curves of the variation of applied magnetic field versus induced field in alloy 1 $\left(\mathrm{Co}_{67.5} \mathrm{Fe}_{3.5} \mathrm{Si}_{17.4} \mathrm{~B}_{11.6}\right)$, under alternate fields, in the as-cast and stress annealed conditions, respectively.

The variations in the values of coercive force, remanent induction and saturation fields of alloy 1 obtained from the hysteresis curve in as-cast and stress annealed conditions are shown in Table 3 . Note that the treated alloy requires a much larger field, Hs, to become saturated than do the untreated alloys. At the same, time its saturation induction, Bs, is much lower than that of untreated alloys. These alterations are due to the phenomenon of hysteresis curve rotation. The treated sample also showed a reduction of the coercive force value, suggesting an improvement in the resolution of signals through a reduction

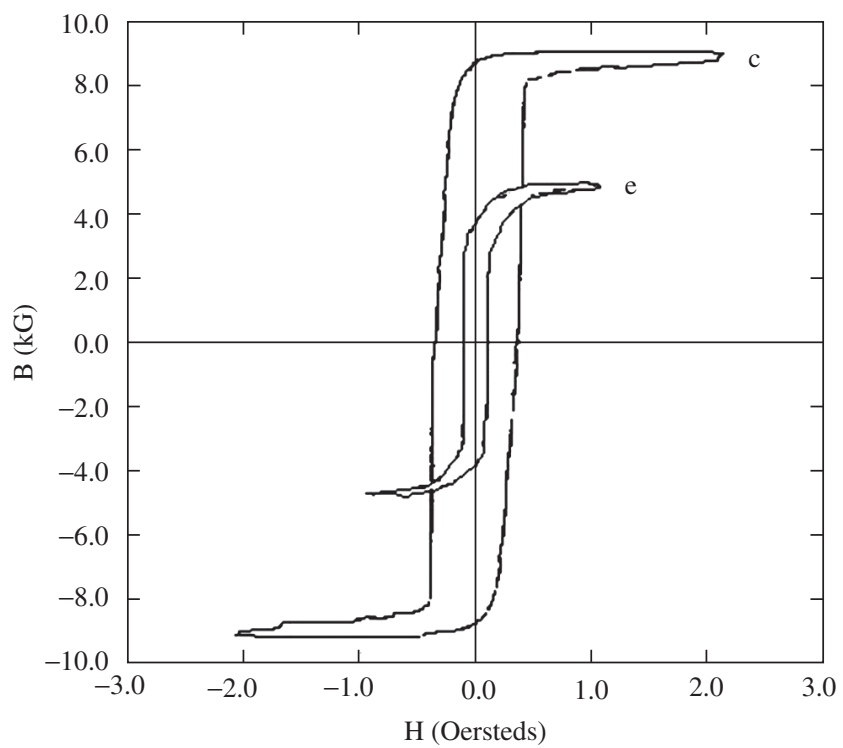

Figure 3. Hysteresis curves of as-cast samples $\mathrm{C}$ and $\mathrm{E}$, at a frequency of $10 \mathrm{kHz}$.

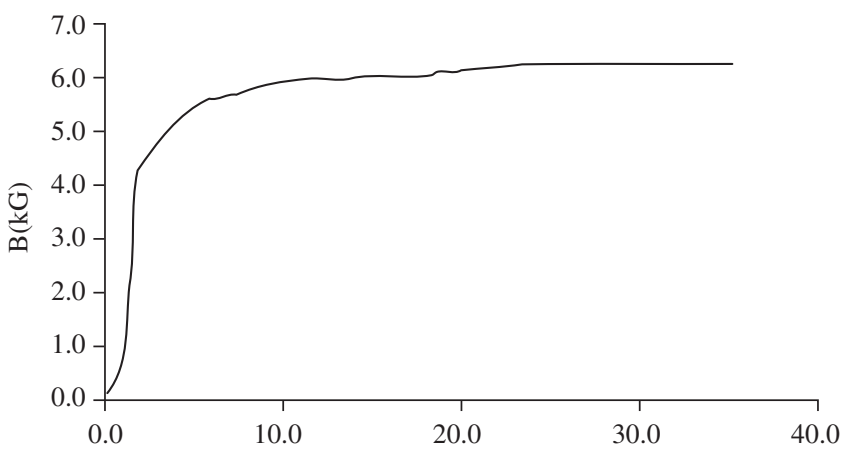

Figure 4. Representative curve of the variation of applied field vs. induced field, of alloy $\mathrm{Co}_{67.5} \mathrm{Fe}_{3.5} \mathrm{Si}_{17.4} \mathrm{~B}_{11.6}$, in the as-cast condition, at a frequency of $7.5 \mathrm{kHz}$.

Table 3. Magnetic properties of the alloys obtained from the hysteresis curve in the as-cast and stress annealed conditions in alloy 1 , with $\mathrm{f}=7.5 \mathrm{kHz}$.

\begin{tabular}{cccccrr}
\hline Samples & $\mathrm{Hs}(\mathrm{Oe})$ & $\mathrm{Bs}(\mathrm{kG})$ & $\mathrm{Hc}(\mathrm{Oe})$ & $\mathrm{Br}(\mathrm{kG})$ & $\mathrm{Hs} / \mathrm{Hc}(\%)$ & $\mathrm{Bs} / \mathrm{Br}(\%)$ \\
\hline Untreated & 0.43 & 5.31 & 0.23 & 4.40 & 188.02 & 120.68 \\
Treated & 3.52 & 2.52 & 0.14 & 0.11 & $2,514.29$ & $2,290.91$
\end{tabular}

of the noise level. The sharp decline in the remanent induction value was due to the major reduction of the curve area concomitant with its rotation.

A comparative study was also done of the material's inductance variation and the applied field. The results are presented in Figures 6 and 7 for the as-cast and stress annealed conditions, respectively. At the end of the linear area of the hysteresis, where the permeability is the highest (at the beginning of the graph, Figure 7), there is a peak in the inductance value which corresponds to the moment of the applied field saturation. After the saturation, the curve shows a continual decline to low values of inductance, near zero. This behavior occurs in parallel with a decrease in the material's

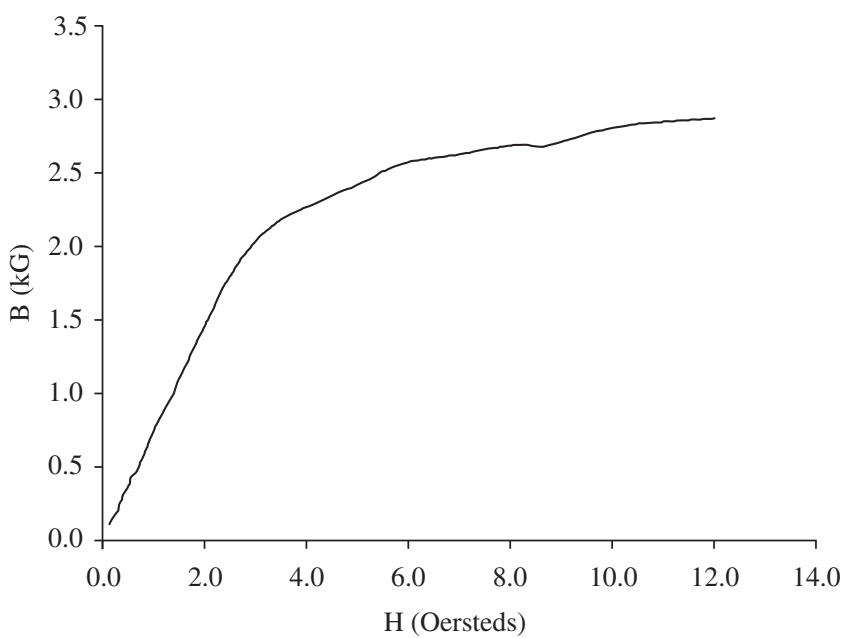

Figure 5. Representative curve of the variation of field applied versus induced field, of alloy $\mathrm{Co}_{67.5} \mathrm{Fe}_{3.5} \mathrm{Si}_{17.4} \mathrm{~B}_{11.6}$, after stress annealing preceded by stress relief, at a frequency of $7.5 \mathrm{kHz}$.

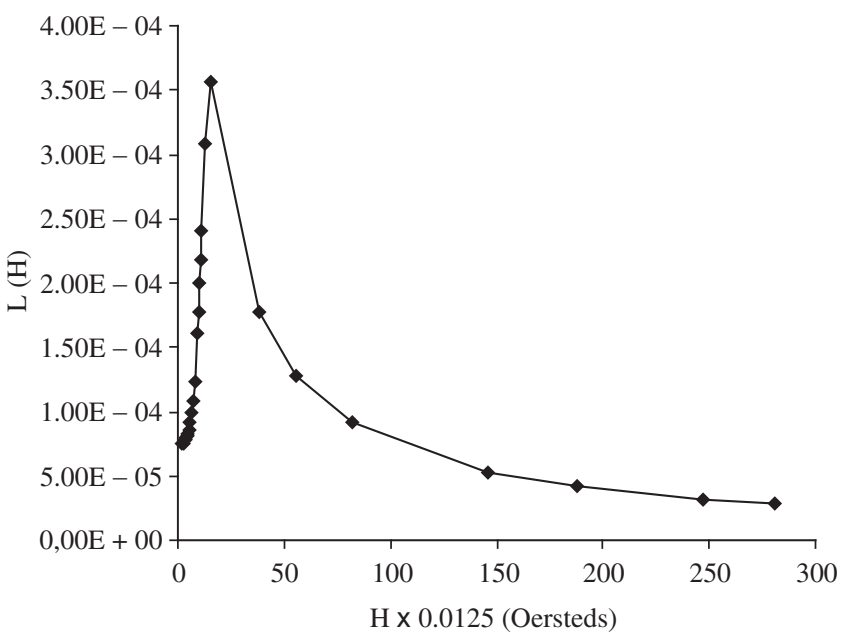

Figure 6. Variation of inductance in relation to the applied field of as-cast alloys, at a frequency of $7.5 \mathrm{kHz}$. 


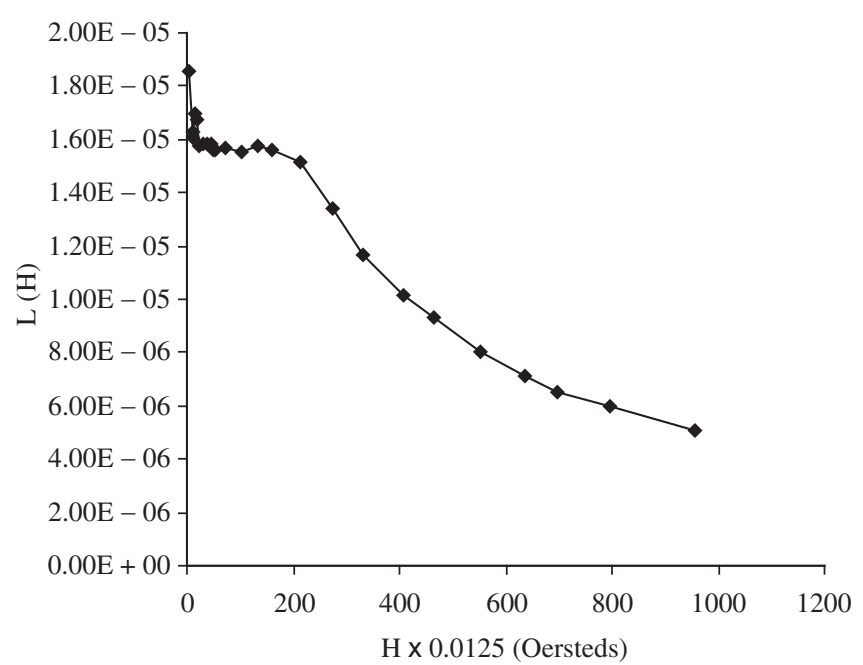

Figure 7. Variation of inductance in relation to the applied field of stress annealed alloys, at a frequency of $7.5 \mathrm{kHz}$.

permeability. The same behavior is illustrated in Figure 7 for the treated material, albeit on a different scale. This comparative study was carried out at a frequency of $7.5 \mathrm{kHz}$.

An analysis was also made of the influence of annealing and type of alloy on the loss of hysteresis energy. Table 2 presents the values of power losses, or energy, by the hysteresis cycle of samples throughout the frequency interval studied here. Alloys 1 and 3 presented the lowest values of energy loss by hysteresis cycle. However, alloy 1 showed the best results. After stress annealing, the low coercive force and remanent induction values were reduced to an even lower hysteresis curve energy loss, allowing for low noise levels in this alloy applied in fluxgate sensor cores.

\section{Conclusions}

No significant variation was observed in the coercive forces of samples of the same alloy with different internal diameters, in spite of the different stresses introduced by the various curvature radii imposed on each sample. On the other hand, the highest internal stress generated in the ribbon of smaller curvature radius produced a higher value of saturation induction in relation to the larger diameter sample, causing an increase in the loss of energy by hysteresis and by eddy currents. The greater stress observed in the sample ribbon of smaller diameter prevented a pronounced rotation of magnetic domains as a consequence of the hysteresis curve, rendering the material less appropriate for use in fluxgate magnetometers.

The magnetic properties of the samples showed no significant differences when different materials were used in the preparation of the core support, i.e., Titanium and Nylon, for a fluxgate magnetometer.

Large widths imposed on ribbons at the moment of their production caused the material to lose a significant amount of energy by the Joule effect and significantly increased its remanence.

Stress annealing induced the appearance of a transverse magnetic anisotropy, causing rotation of the spontaneous domains of magnetization, which is congruent with previous studies. The hypothesis of Nielsen et al. confirms the importance of the longitudinal axis of the amorphous ribbon as an easy magnetization axis, as well as the need for a convenient rotation of domain walls to achieve low noise levels in fluxgate sensors. Stress annealing the $\mathrm{Co}_{67.5} \mathrm{Fe}_{3.5} \mathrm{Si}_{17.4} \mathrm{~B}_{11.6}$ alloy allows one to produce fluxgate sensors with lower noise levels than those of commercial crystalline materials.

\section{Acknowledgements}

The authors gratefully acknowledge FAPERJ (Brazil) for its partial financial support of this work. We are also indebted to the Physics Institute of USP and to Dr. Tsuneharu Ogasawara (COPPE/ UFRJ) for the use of their facilities, and to V. M. Hamza and A. R. R. Papa for their relevant comments. The authors sincerely thank the anonymous reviewer for his corrections, suggestions and criticism, which we consider, have remarkably improved the presentation of this work.

\section{References}

1. Kubik J, Pavel L, Ripka P, Kaspar P. Low-Power Printed Circuit Board Fluxgate Sensor. IEEE Sensors Journal 2007; 7(2):179-183.

2. Nielsen OV. [personal communication] 1993.

3. Benyosef LCC, Teodósio JR, Taranichev VE, Jalnin BV. Improvements on $\mathrm{CoFeSiB}$ Amorphous Ribbon for Fluxgate Sensor Cores. Scripta Metallurgica et Materialia 1995; 33(9):1451-1454.

4. Nielsen OV, Petersen JR, Fernandez A, Hernando B, Spisak P, Primdahl F, Moser N. Analysis of a fluxgate magnetometer based on metallic glass sensors. Measurement Science \& Technology 1991; 2(5):435-440.

5. Shirae K. Noise in Amorphous Magnetic Materials. IEEE Transactions on Magnetics 1984; 20(5):1299-1301.

6. Gibbs MRJ. Anisotropy and Magnetostriction in Amorphous Alloy. Journal of Magnetism and Magnetic Materials 1990; 83(1-3):329-333.

7. Ripka P. Improved Fluxgate for Compasses and Positions Sensors. Journal of Magnetism and Magnetic Materials 1990; 83(1-3):543-544.

8. Záveta K, Nielsen OV, Jurek K. A Domain Study of Magnetization Processes in A Stress annealed Metallic Glass Ribbon for Fluxgate Sensor. Journal of Magnetism and Magnetic Materials 1992; 117(1-2):61-68.

9. Taranichev VE, Alenov MN, Nemova OYU. Magnetization of Amorphous Alloys by Elastic Strain. Journal of Magnetism and Magnetic Materials 1994; 131(1-2):229-234.

10. Zbroszczyk J, CiurzyImagenska W, WysImageiocki B, Szymura S, Swierczek J, Yamashiro Y, Narita K. Effect of External Tensile Stress on Some Magnetic Properties of Rapidly Quenched Ribbons. Journal of Magnetism and Magnetic Materials 1992; 112(1-3):341-343.

11. Záveta K, Nielsen OV, Jurek K. A domain study of magnetization processes in a stress annealed metallic glass ribbon for fluxgate sensors. Journal of Magnetism and Magnetic Materials 1992; 117(1-2):61-68.

12. Ziewiec K, Olszewski P, Gajerski R, Jakub-Michalski J, Konopka K. Amorphization and thermal stability of $\mathrm{Cu}_{68_{5} 5} \mathrm{Ni}_{12} \mathrm{P}_{19} \cdot{ }_{5}$ alloy. Journal of Alloys and Compounds 2004; 384(1-2):175-180.

13. Hasegawa R. Applications of amorphous magnetic alloys. Materials Science and Engineering A 2004; 375-377:90-97

14. Liu XB, Liu XD, Altounian Z, Tu GH. Phase formation and structure in rapidly quenched $\mathrm{La}\left(\mathrm{Fe}_{0 \cdot 88} \mathrm{Co}_{0 \cdot{ }_{12}}\right)_{13 ?} x \mathrm{Six}$ alloys. Journal of Alloys and Compounds 2005; 397(1-2):120-125.

15. Primdahl F, Ripkat $P$, Petersen JR, Nielsen OV. The sensitivity parameters of the short-circuited fluxgate. Measurement Science \& Technology 1991; 2(11):1039-1045.

16. Zhukov A, Zhukova V, Larin V, Gonzalez J. Tailoring of magnetic anisotropy of Fe-rich microwires by stress induced anisotropy. Physica B 2006; 384(1-2):1-4.

17. Gunther W. Stress annealing process suitable for the production of low permeable nanocrystalline tape wound cores. Journal of Magnetism and Magnetic Materials 2005; 290-291(Part 2):1483-1486.

18. Kraus L. Magnetoelastic hysteresis of amorphous ribbons. Journal of Applied Physics 2003; 93(10):7220-7222.

19. Benyosef LCC, Teodosio JR, Taranichev VE, Zhalnin BV, Nemova OYU. Effect of Stresses on the Magnetic Properties of Amorphous Ribbons for Fluxgate magnetometers. Journal of Advanced Materials 1996; 3(2):140-143.

20. Vázquez M, Fernengel W, Kronmüller $H$. The effect of tensile stresses on the magnetic properties of $\mathrm{Co}_{58} \mathrm{Fe}_{5} \mathrm{Ni}_{10} \mathrm{Si}_{11} \mathrm{~B}_{16}$ amorphous alloys. Physica Status Solidi (a) 1983; 80(1):195-204. 